\author{
dspace.vutbr.cz
}

\title{
Characteristics of Turbulent Particle Transport in Human Airways under Steady and Cyclic Flows
}

\author{
JEDELSKÝ, J.; LÍZAL, F.; JÍCHA, M.
}

International Journal of Heat and Fluid Flow

2012, vol. 35, June 2012, pp. 84-92

ISSN: 0017-9310

DOI: https://doi.org/10.1016/j.ijheatfluidflow.2012.01.003

Accepted manuscript

(C) 2012. This manuscript version is made available under the CC-BY-NC-ND 4.0 license (http://creativecommons.org/licenses/by-nc-nd/4.0/), doi:

https://doi.org/10.1016/j.ijheatfluidflow.2012.01.003

Final version available from https://www.sciencedirect.com/science/article/pii/s0142727X12000045 


\title{
CHARACTERISTICS OF TURBULENT PARTICLE TRANSPORT IN HUMAN AIRWAYS UNDER STEADY AND CYCLIC FLOWS
}

\author{
Jan Jedelsky, Frantisek Lizal and Miroslav Jicha \\ Faculty of Mechanical Engineering \\ Brno University of Technology \\ Technicka 2896/2, 61669 Brno, Czech Republic \\ jedelsky@fme.vutbr.cz; lizal@fme.vutbr.cz; jicha@fme.vutbr.cz
}

\begin{abstract}
Motion of monodispersed aerosol particles suspended in air flow has been studied on realistic transparent model of human airways using Phase Doppler Particle Analyser (P/DPA). Time-resolved velocity data for particles in size range 1 to $8 \mu \mathrm{m}$ were processed using Fuzzy Slotting Technique to estimate the power spectral density (PSD) of velocity fluctuations. The optimum processing setup for our data was found and recommendations for future experiments to improve PSD quality were suggested. Typical PSD plots at mainstream positions of the trachea and the upper bronchi are documented and differences among (1) steady-flow regimes and equivalent cyclic breathing regimes, (2) inspiration and expiration breathing phase and (3) behaviour of particles of different sizes are described in several positions of the airway model. Systematically higher level of velocity fluctuations in the upper part of the frequency range $(30-500 \mathrm{~Hz})$ was found for cyclic flows in comparison with corresponding steady flows. Expiratory flows in both the steady and cyclic cases produce more high-frequency fluctuations compared to inspiratory flows. Negligible differences were found for flow of particles in the inspected size range 1 to $8 \mu \mathrm{m}$ at frequencies below $500 \mathrm{~Hz}$. This finding was explained by Stokes number analysis. Implied match of the air and particle flows thereby indicates turbulent diffusion as important deposition mechanism and confirms the capability to use the P/DPA data as the air flow velocity estimate.
\end{abstract}

\section{INTRODUCTION}

Transport and deposition of aerosol in human airways has been of research interest for several decades. Main present motivation for elucidation of related phenomena is the increasing tendency of therapeutical drug application in the form of the inhaled aerosol (Azarmi et al., 2008). Aerosol deposition in the human airways is also extensively studied in many industrial hygiene related researches (Kleinstreuer et al., 2008; Sosnowski et al., 2007; Su and Cheng, 2009 and others).

Published works show that the air flow in the multiplebifurcating airway system is a very complex phenomena showing turbulent, transitionary and laminar behaviour depending on breathing conditions, morphology and position in the airways (Cohen et al., 1993; Guan and Martonen, 2000;
Ramuzat and Riethmuller, 2002; Martonen et al., 2002; Kleinstreuer and Zhang, 2003; Fresconi et al., 2003; Li et al., 2007). Transitionary and turbulent flows often occur in the mouth-throat and upper conducting airways while laminar flow controls transport beyond approximately the 9th generation of the bronchi. Within this wide range of conditions, fate of the inhaled particles depends on their size in conjunction with the local flow dynamics. Aerosol deposition may occur due to impaction, sedimentation, diffusion, and turbulent dispersion.

The occurrence of upstream turbulent flows may influence both the inlet velocity and particle profiles entering the bifurcation and may enhance deposition within the model (Longest and Holbrook, 2011). The turbulence induced by the laryngeal jet significantly affects airway flow patterns as well as tracheal wall shear stress (Lin et al., 2007) and is responsible for increased local particle deposition (Gemci et al., 2002; Chan et al., 1980). Kleinstreuer and Zhang (2003) demonstrated the importance of transitional and turbulent flows on particle deposition throughout an oral-trachea airway model. They reported enhanced particle deposition in the trachea near the larynx due to turbulence and throughout the airway mainly for small particles $(S t k<0.06)$ due to turbulent dispersion. Sosnowski et al., 2007 used CFD modelling to study behaviour of aerosol particles with size $0.3-10 \mu \mathrm{m}$ in the oro-pharynx under unsteady airflow and found higher deposition efficiency for smaller particles due to strong effects of turbulent diffusion. Kleinstreuer et al., 2008 modelled deposition of micron-size particles in pulmonary airway replicas and located particles mainly around the carinal ridges deposited due to inertial impaction but some particles also landed outside the vicinities of the cranial ridges due to secondary flows and turbulent dispersion. Cheng et al., 1997 used oral airway replicas and found that turbulent diffusional deposition is the dominant deposition mechanism of ultrafine particles $(<0.1 \mu \mathrm{m})$.

Turbulence in the respiratory tract is generated by several mechanisms. Primary source of turbulence is often the inhaler, which delivers particles into the mouth producing high momentum turbulent spray jet. A high-speed laryngeal jet is formed as the flow passes through the glottis; this jet induces turbulent flow in the trachea. Lin et al. (2007) found out that regions of maximum local turbulence in the trachea are associated with Taylor-Görtler-like coherent vortical 
structures in the supraglottis and the subglottis. Hiemenz flow (characterised by transition to turbulence at low Reynolds numbers (250) (Obrist et al., 2011)) at the carinal ridges can be related to the increased turbulence in daughter branches during inspiration. Overall fluid motion in curved airway tubes has helical character (Guan and Martonen, 2000); the vortices formed contribute to turbulence. It was confirmed in the left curved bronchus by Große et al. (2007). Mixing of streams from daughter branches is a source of turbulence for expiratory flows. Complex flow structures containing different types of vortices, flow detachment, wakes, simultaneous bidirectional flow, recirculation zones and velocity oscillations observed particularly for cyclic breathing as described in a number of papers (brief review in Jedelsky et al., 2010a) contribute to the turbulence as well. The turbulence in airways is generally strongly anisotropic with major part of turbulent kinetic energy (TKE) contained preferably in axial direction of the flow (Longest and Holbrook, 2011).

Turbulent flow contains unsteady vortices which appear on many different length scales and interact with each other. Most of the kinetic energy of the turbulent motion is contained in the large-scale structures generated by the flow. These energetic structures transform to smaller-scale vortices by an inertial and basically inviscid mechanism. Smaller and smaller structures are produced until they are small enough for molecular diffusion to become important and viscous dissipation of energy finally takes place. This process known as the energy "cascade" leads to essentially continuous frequency spectrum of TKE. The largest eddies appear at low frequencies and are followed by energy containing eddies that are characterized by maximum amplitude at the spectrum. Inertial subrange, at higher frequencies, shows decreasing TKE tendency with frequency and successive dissipation subrange at the highest frequencies shows even faster TKE decay with frequency. The TKE spectrum can be estimated using time-resolved measurement of velocity of particle laden turbulent flows by laser-Doppler techniques such as P/DPA. Fate of airborne particles in turbulent flows depends on their $S t k$. Very small particles $(S t k<1)$ follow the fluid motion, while larger particles $(S t k \sim 1)$ tend to be centrifuged toward the peripheries of the vortical structures. For Stk $>1$ the particles move essentially independent of the fluid (Crowe et al., 1998). Small Stk particles, transported by turbulent eddies, can deposit if forced by these eddies towards the airway wall. Particles with $S t k \sim 1$, concentrated at the peripheries of turbulent structures (Zhang and Kleinstreuer, 2002), can deposit as well. Turbulent dispersion is therefore responsible for increased deposition efficiency.

Continuous movement from simple airway models and steady flows to realistic models and lifelike cyclic flow regimes is seen on present CFD simulations and experimental studies. Realistic, CT based, lung models were proved to be a must for valid results of flow and particle transport. Several works report significant quantitative and qualitative differences between aerosol transport/deposition characteristics under steady flows, most frequently studied in

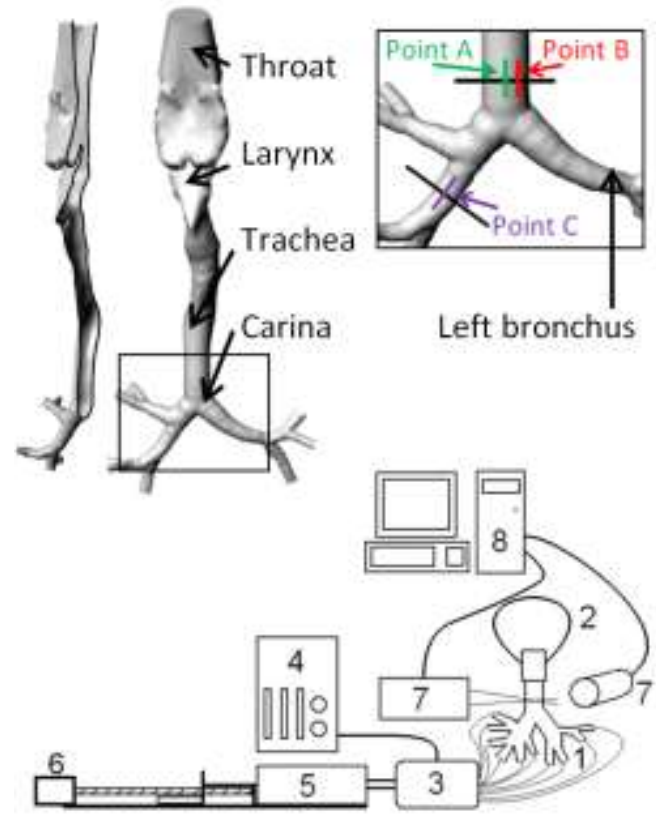

Figure 1. The airway model with measurement positions in expanded window (top). A schematic diagram of the test rig (bottom). (1) the airway model, (2) a collecting sac, (3) mixing chamber, (4) condensation monodisperse aerosol generator, (5) pneumatic cylinder with piston, (6) computer controlled step motor with motion screw, (7) P/DPA, and (8) PC.

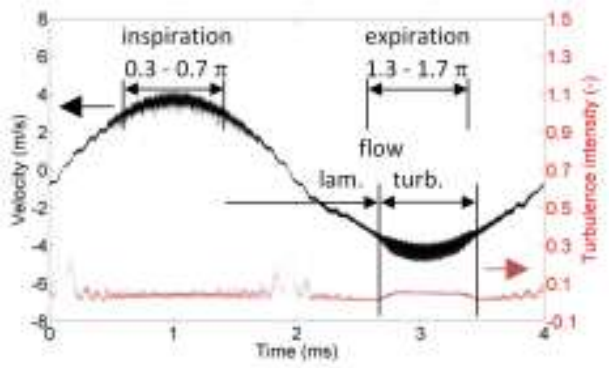

Figure 2. Ten overlapped (phase-averaged) cycles. Inspiratory and expiratory time windows used for PSD estimations are marked. Sections with laminar and turbulent character of flow during the expiratory phase are distinguished.

the past, and lifelike oscillatory flows (Lieber and Zhao, 1998; Zhang and Kleinstreuer, 2004; Zhang et al., 2001).

Several methods have been applied for flow studies in airway models. Intrusive Hot Wire Anemometry (HWA) was used for in vitro air flow measurements in the past while optical methods prevail today. The most common is Particle Image Velocimetry (PIV). Laser Doppler Anemometry (LDA) (Corieri and Riethmüller, 1989; Lieber and Zhao, 1998; Tanaka et al., 1999; Corcoran and Chigier, 2000) and P/DPA 
(Gemci et al., 2002) are less frequent. Complex design of realistic models hampers application of optical methods for aerosol transport studies. PIV, in realistic models, requires application of a liquid with refraction index equal to the one of the model walls instead of air, as the real breathed fluid, or usage of simple models with cylindrical walls if air used. LDA (or P/DPA), as point-wise technique is more suitable for measurements in the composite optical system of transparent model with flowing air. This method moreover allows direct particle flow measurement with high spatial resolution and high data rate. Aside basic flow characteristics as mean and rms velocities also spectral flow properties could be estimated therefore. Estimation of PSD from Laser-Doppler based data faces a problem of irregular time sampling. A number of different techniques was developed to treat this problem; it was formerly solved using analogue filters of the velocity signals or by equidistant time re-sampling. Scott (1974) derived an expectation of the spectrum on the basis of a Slot Correlation technique (SC) which remains even today one of the most viable means for PSD estimations. Large group of estimators is based on the concept of signal reconstruction and re-sampling at equal time intervals. The most common reconstruction is zeroth-order interpolation or sample and hold $(\mathrm{S}+\mathrm{H})$ method. Other spectral estimator for LDA data, introduced by Nobach et al. (1996), was based on one-point reconstruction techniques employing a refinement which accounts, in a statistical manner, for the velocity change between a particle arrival and the sample instant. The most reliable spectral estimation techniques are explained in detail by Benedict et al. (2000).

In this work, we have focused on description of the turbulence in frequency domain for the case of the complex flow in the multiple bifurcating airways, which is not sufficiently studied topic so far. We have processed exemplary P/DPA data acquired during our earlier study ${ }^{1}$ with the aim to document spectral properties of turbulent particle transport in human airways. Brief description of our experiment and measured data is made first. Kern software program (Nobach, 2002) is used for estimation of PSD of particle velocity fluctuations. Setup of the program has been optimized for our data and several P/DPA data sets are processed. Arbitrary PSD plots are shown to describe nature of the particle-air flow. Differences between steady and cyclic flows at several positions of the airway model and the influence of the flow regime and particle size on the PSD are discussed. Several recommendations for future to improve the PSD estimations are given.

\section{EXPERIMENTAL APPARATUS}

Our experimental device (Fig. 1) uses a computer controlled motor (6) which drives piston through pneumatic

\footnotetext{
${ }^{1}$ This study of transport of monodispersed micron-sized liquid particles dispersed in air was made in realistic transparent human airway model for a range of steady and cyclic flows and particles of various sizes. P/DPA was used to acquire time-resolved data of particle velocity (Jedelsky et al., 2010a).
}

cylinder (5) as a source of oscillating air flow. Steady air flows were maintained using a downstream suction source vacuum air pump. Three steady breathing regimes $(15,30$ and 60 litre/min) and three corresponding cyclic sinusoidal breathing regimes were used (tidal volume 0.5 litre and breathing period $4 \mathrm{~s}, 1$ litre \& $4 \mathrm{~s}$ and 1.5 litre \& $3 \mathrm{~s}$ ). Monodispersed aerosol particles of di-2-ethyl hexyl sebacate (DEHS) ranging from 1 to $8 \mu \mathrm{m}$ are generated by condensation generator (4). One-half of the particles is mixed with the air in a chamber (3) using static mixer and flows into the airway model (Fig. 1). The second half flows into a sac (2), that collects the particle-air mixture for the second breathing cycle phase. The particle-laden air flow is incompressible, subsonic, isothermal, and viscous, with high particle/air density ratio of 760 and the DEHS aerosol is nonevaporating/non-condensing. 1D P/DPA by Dantec Dynamics (7) was used for measurement of the particle size and mainly for time-resolved measurement of the axial velocity component of the particle motion during breathing cycle in several cross-sections of the model ${ }^{2}$.

A transparent thin-walled human airway model with realistic geometry was used for the investigation. The airway geometry was captured using high resolution CT scan of an adult Caucasian male. The model, made of transparent silicon Sylgard 184 (Dow Corning), covers airways from midpharynx to $3^{\text {rd }}-4^{\text {th }}$ generation of bronchi, it preserves realistic shapes of the airways with complex structures of glottis and epiglottis and 3-D asymmetric branching as seen in Fig. 1, top. Mean tube diameters in each generation are documented in Table 1. Geometry and fabrication of the model is described in detail by Lizal et al., 2011, flow rate distribution into particular branches of the tracheobronchial tree for different steady and cyclic breathing regimes is documented in (Jedelsky et al., 2009). The model was placed in upstanding human position and it was fixed in a frame mounted to a traversing mechanism for positioning during experiments.

Thin model walls are required for optical access into the model without strong optical distortions when the air flow or particle transport with air as a carrier medium to be studied. This approach differs from other fluid dynamics experiments in complex airway models, where liquids with the refraction index matched to the one of the model material are used (Ramuzat and Riethmuller, 2002; Fresconi and Prasad, 2007; Große et al., 2007; Adler and Brücker, 2007; Theunissen and Riethmuller, 2008). The realistic transparent airway model is asymmetric, it has non-circular cross-sections, walls of varying thickness and varying curvature. Optical measurement in this model requires very precise setup and approach different to the one used for measurement with simple optical path. It is necessary to adjust the laser beam crossing every time the measurement position changes. An accurate choice of the measurement position is needed to avoid strong distortion

\footnotetext{
${ }^{2}$ P/DPA was chosen instead of simpler LDA to verify particle size and also to reduce unwanted sources of noise in PSD estimation such as reflections and multiple-particle scattering. For more information on the P/DPA setup and data processing see Jedelsky et al. (2010a).
} 
of the laser beam even when thin-wall model used. Measurement in near-wall regions of the model faces stronger optical distortion and therefore main-stream positions are preferred to guarantee good results.

\section{RESULTS AND DISCUSSION}

Table 1 documents basic flow characteristics in the human model and shows variation of Reynolds $(R e)$, Stokes $(S t k)$ and Womersley $(\alpha)$ numbers $^{3}$ with flow rate $\bar{Q}$ and position in the airways. The $\bar{Q}$ values used for steady flows correspond to rms flow rate values during cyclic flows. The tracheobronchial tree is numbered according to Weibel $(0=$ trachea, $\mathrm{L}=$ larynx). Average tube diameter, $\bar{D}$, in particular generation is taken from Schmidt et al. (2004) and represents the average value over all branch diameters within the generation. The larynx diameter was defined as a hydraulic diameter in the location of the smallest cross-sectional area between mid-pharynx and trachea. $R e$ is proportional to the mean flow velocity $\bar{v}$ and it decreases as tube diameter, $\bar{D}$, drops down. Only several combinations for Stk were calculated: the smallest particle size \& minimal flow rate, medium particle size \& medium flow rate and the largest particles \& maximal flow rate. Maximum Stk about 0.1 was found in the second generation for $8 \mu \mathrm{m}$ particles and flow rate 60 1/min; for all other cases usually $S t k \ll 1$.

Exemplary results of the two-phase air-particle flow under cyclic breathing are illustrated in Fig. 2. The measurement was performed at point $\mathrm{C}$ (Bronchus Intermedius, centreline, see Fig. 1) for flow regime 1 litre $\& 4 \mathrm{~s} ; 4 \mu \mathrm{m}$ particles were used. The time-resolved axial velocity of individual aerosol particles passing through the measurement volume of P/DPA as well as the axial turbulence intensity are displayed in the plot. We assume that particles follow the air flow with no significant slip due to their low $S t k$ and discus this assumption in chapter Stokes number in detail. Particle velocity data are thus used for air velocity and turbulence intensity estimation. It is seen that the particle velocity during the cyclic flow approximately corresponds to the sinusoidal shape of the cyclic breathing. The positive velocity values stand for inspiration, negative stand for expiration.

Extensive documentation of further results of the particle transport supported with explanation of the effect of various factors (particle size, steady/cyclic flows, spatial and temporal nature of the flow) was already made in (Jedelsky et al., 2010b). The average axial turbulence intensities at point $\mathrm{A}$ (placed in trachea centreline, $20 \mathrm{~mm}$ above carina, see Fig. 1) at steady and cyclic inspiration flows are compared in Table 2 to illustrate typical results of these basic flow characteristics. Small differences between both the cases and also among different flow rates and particle sizes were found. Turbulence intensity covers range $0.07-0.11$ for all the cases and no

\footnotetext{
${ }^{3}$ All the dimensionless numbers are calculated for the average diameter, $\bar{D}$, as characteristic length scale of given tube.
}

significant correlation between the flow regime and the turbulence intensity is seen. It is interesting as $R e$ varies significantly: from 1200 to 4800 (see Table 1). The relatively high and regime independent turbulence intensity can be attributed to the complexity of the upstream geometrical structures of larynx.

Table 1: Dimensionless numbers in individual branching levels.

\begin{tabular}{cccccccc}
\hline \hline Gen. & $\bar{Q}$ & $\bar{D}$ & $\bar{v}$ & $d_{p}$ & & & \\
& $(1 / \mathrm{min})$ & $(\mathrm{mm})$ & $(\mathrm{m} / \mathrm{s})$ & $(\mu \mathrm{m})$ & & $R e$ & $\alpha$ \\
\hline \multirow{3}{*}{$\mathrm{L}$} & 15 & & 1.34 & 1 & $3.32 \mathrm{E}-04$ & 1368 & 5.0 \\
& 30 & 15.4 & 2.68 & 4 & $9.75 \mathrm{E}-03$ & 2736 & 5.0 \\
& 60 & & 5.37 & 8 & $7.69 \mathrm{E}-02$ & 5472 & 5.7 \\
\hline \multirow{3}{*}{0} & 15 & & 1.05 & 1 & $2,30 \mathrm{E}-04$ & 1211 & 5.6 \\
& 30 & 17.4 & 2.10 & 4 & $6,76 \mathrm{E}-03$ & 2421 & 5.6 \\
& 60 & & 4.21 & 8 & $5,33 \mathrm{E}-02$ & 4843 & 6.5 \\
\hline \multirow{3}{*}{1} & 15 & & 1.18 & 1 & $3,90 \mathrm{E}-04$ & 908 & 3.7 \\
& 30 & 11.6 & 2.37 & 4 & $1,14 \mathrm{E}-02$ & 1816 & 3.7 \\
& 60 & & 4.73 & 8 & $8,89 \mathrm{E}-02$ & 3632 & 4.3 \\
\hline \multirow{3}{*}{2} & 15 & & 1.16 & 1 & $5,40 \mathrm{E}-04$ & 635 & 2.7 \\
& 30 & 8.3 & 2.31 & 4 & $1,56 \mathrm{E}-02$ & 1269 & 2.7 \\
& 60 & & 4.62 & 8 & $1,21 \mathrm{E}-01$ & 2538 & 3.1 \\
\hline \multirow{3}{*}{3} & 15 & & 0.75 & 1 & $4,00 \mathrm{E}-04$ & 361 & 2.4 \\
& 30 & 7.3 & 1.49 & 4 & $1,14 \mathrm{E}-02$ & 721 & 2.4 \\
& 60 & & 2.99 & 8 & $8,89 \mathrm{E}-02$ & 1443 & 2.7 \\
\hline \multirow{3}{*}{4} & 15 & & 0.66 & 1 & $4,60 \mathrm{E}-04$ & 239 & 1.8 \\
& 30 & 5.5 & 1.32 & 4 & $1,33 \mathrm{E}-02$ & 479 & 1.8 \\
& 60 & & 2.63 & 8 & $1,05 \mathrm{E}-01$ & 958 & 2.0 \\
\hline \hline
\end{tabular}

Table 2. Axial turbulence intensity at point A.

\begin{tabular}{ccccccc}
\hline \hline \multirow{2}{*}{$\begin{array}{c}\text { Size } \\
(\mu \mathrm{m})\end{array}$} & \multicolumn{3}{c}{$\begin{array}{c}\text { Steady Inspiration } \\
\text { (litre/min) }\end{array}$} & \multicolumn{2}{c}{ Cyclic Inspiration } \\
\cline { 2 - 7 } & 15 & 30 & 60 & $0.5 \& 4^{5}$ & $1.0 \& 4$ & $1.5 \& 3$ \\
\hline 1 & 0.104 & 0.085 & 0.071 & $\mathrm{NaN}$ & 0.092 & $\mathrm{NaN}$ \\
4 & 0.107 & 0.110 & 0.091 & 0.095 & 0.097 & 0.084 \\
8 & 0.109 & 0.092 & 0.074 & $\mathrm{NaN}$ & 0.095 & $\mathrm{NaN}$ \\
\hline \hline
\end{tabular}

The probability density function (PDF) of the particle velocity was evaluated for steady flow conditions $30 \mathrm{l} / \mathrm{min}$ at point A. The particle velocity samples were classified into

\footnotetext{
${ }^{4}$ The turbulence intensity in cyclic inspiration flow is calculated as an average value during a phase window $0.3-0.7 \pi$ (see Fig. 2) averaged for ten consequent cycles.

${ }^{5}$ Tidal volume (litre) \& period of cycle ( $\mathrm{sec}$ )
} 
bins with size of $0.15 \mathrm{~m} / \mathrm{s}$. Velocity counts in each bin were then divided by the total sample number to give the relative counts displayed in Fig. 3. The velocity distribution corresponds to log-normal distribution with moderate inclination of the maximum to higher velocities. This bias is caused by specific P/DPA sampling rate; where for spatially uniform particle concentration the data rate is proportional to actual flow velocity (see Eq. (2) bellow). Different particle sizes give similar PDF shapes with no significant systematic differences. As seen above, neither the mean value of turbulence intensity (Table 2) nor the PDF of the particle velocity can resolve differences in flow for different flow regimes and particles of different sizes.

\section{Estimation of PSD of Velocity Fluctuations}

Laser-Doppler based techniques provide flow velocity data with comparatively high temporal resolution so they can serve for estimation of the moments and spectra of the turbulent velocity fluctuations. P/DPA is a tracer-based method with irregular particle arrival times. Presuming an equal particle distribution in space with constant concentration and mean data rate of the measurement $\dot{n}$, the intervals $\Delta t$ between the particles are distributed exponentially: $p(\Delta t)=\dot{n} \cdot e^{-\dot{n} \Delta t}$, as seen in Fig. 11 bellow. The most probable interparticle arrival time is zero so information about very high frequency fluctuations is contained in the data. Nevertheless maximum reliable frequency is $f_{\max } \cong \dot{n} / 2 \pi$ according to Adrian and Yao (1987). The irregularity of the sampling time caused by naturally seeded particles in flow brings difficulties to the estimation of the PSD of the velocity fluctuations.

We employed SC technique for the estimation of the PSD as described by Benedict et al. (2000) and calculated the PSD using Kern software (Nobach, 2002). The software has a number of options and variables to set with no guideline for optimum setup. However resulting PSD is very sensitive to the input parameter values. A large data set was tested to find out the best setup. Published HWA and LDA data of identical flow (Nobach, 2006) were compared first. After successful agreement between these HWA and LDA results we focused on our P/DPA data. A set of three consequent data records was chosen to describe the PSD estimation process. The data were acquired at point $\mathrm{A}$ for steady flow regime $301 / \mathrm{min}$ and $1 \mu \mathrm{m}$ particles. Each file had 16384 samples and measurement period $\sim 5.8 \mathrm{~s}$ (average sampling frequency $2825 \mathrm{~Hz}$ ).

Fig. 4 shows PSD of the velocity fluctuations versus frequency, $f$, using a log-log plot. The average PSD curve based on the three records together with standard deviation was calculated. We have ascertained that any unique software setup does not produce the PSD over the whole frequency range provided by the measured data. So the spectra is composed of two curves; the lower frequency part (3.3 $35 \mathrm{~Hz}$ ) was calculated using: SC, $F=70, K=128, N_{F}=1000$ and standard options $(+$ fil - self $+\mathrm{mbv}+\mathrm{vw}+\mathrm{ln}+\mathrm{fst}+$ fbat + lte

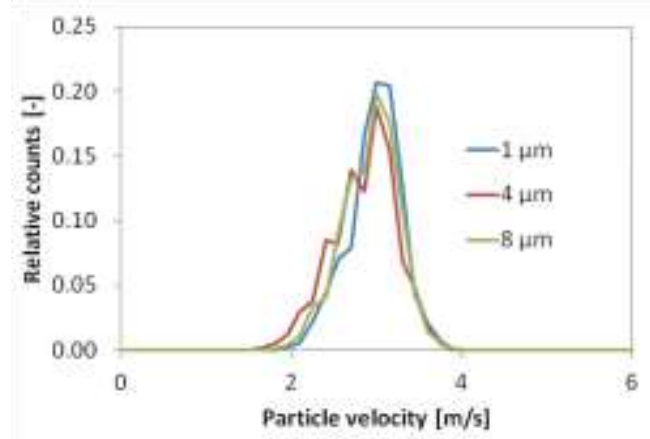

Figure 3. PDF of particle velocity.

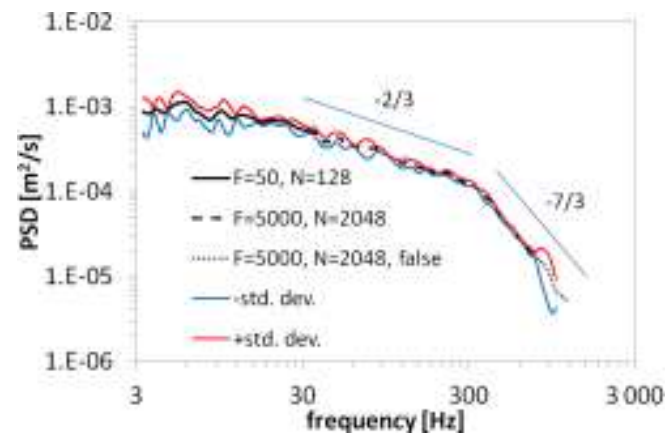

Figure 4. Average PSD with marked standard deviation limits.

$+\mathrm{cft})^{6}$, the upper frequency part was calculated as: SC, $F=$ 5000, $K=2048, N_{F}=1000$ and standard options. Individual PSD plots (not shown here) are very consistent in the frequency range $15-750 \mathrm{~Hz}$ as indicated by the standard deviation curves. Small fluctuations in the amplitude inside this frequency range that were observed in each PSD plot change record to record and therefore are not an inherent property of the flow. Low frequency fluctuations in the PSD amplitude (up to $\sim 15 \mathrm{~Hz}$ ) are a product of natural variations of flow that cannot be fully resolved by the relatively short data record. They lead to differences in particular records and are reflected by increased deviation in Fig. 4. The results differ significantly also for frequencies higher than about 1 $\mathrm{kHz}$ due to lack of useful data from P/DPA measurement. The differences can be partially caused by the processing algorithm as well. This part of the plot is not realistic and cannot be used for analysis.

Variable windowing (vw) (Tummers and Passchier, 1996) used in this calculation leads to smoothening and averaging of fluctuations in individual spectral lines. Larger fluctuations are preserved. The amplitude of velocity fluctuations at the inspected point shows only very mild decreasing tendency with frequency up to about $30 \mathrm{~Hz}$, then slightly higher and relatively constant-slope decrease according $f^{2 / 3}$ in range $30-$

${ }^{6} F$ is the frequency used to define the time lag between samples in the autocorrelation function (ACF), $K$ is the number of samples in the ACF, $N_{F}$ is the number of samples in the spectrum, for better description see Nobach (2002). 
$300 \mathrm{~Hz}$ and more distinct decrease with constant slope $f^{7 / 3}$ for frequencies higher than $300 \mathrm{~Hz}$. No distinct peak in the PSD is obvious. Very similar shape of the PSD was found also at other measurement points, for other particle sizes and other flow regimes in the trachea. The first part of the spectra up to $30 \mathrm{~Hz}$ can be attributed to the energy-containing range, followed by inertial subrange. The exponent $-2 / 3$ at the first part of inertial subrange suddenly changes to $-7 / 3$. This shape of inertial subrange differs from the usual Kolmogorov-like constant value slope $-5 / 3$ due to several reasons. Generated turbulence is anisotropic in large scales due to character of the flow. Energy containing eddies are continually generated during flow in the complex airway geometry. The cumulative TKE was calculated for given data. The amplitude of oscillations at frequencies higher than $800 \mathrm{~Hz}$ was approximated using the $f^{7 / 3}$ rule. Low frequency oscillations up to $140 \mathrm{~Hz}$ participate on $50 \%$ of the total TKE and $90 \%$ is covered in range up to $620 \mathrm{~Hz}$.

The calculation made with the same setup as with the previous results but without vw produced PSD plots containing strong scatter in the amplitude. No significant coherence of this "noise" in spectra among individual records was found. Averaging of the PSD from the three records (Fig. 5) reduces this scatter so processing of multiple data files or long measurements is convenient for statistically correct results. Variable windowing is even stronger instrument for the data smoothening mainly at high frequencies as indicated in the plot.

\section{Steady Flows}

Several P/DPA data sets were used to find out the influence of the flow regime and particle size on the PSD of velocity fluctuations and to describe differences between inspiration and expiration flows and spatial variations of the PSD. The data in main-stream positions showing relatively simple flows without wakes and recirculation zones were used.

Flow Regimes. PSD plots for three inspiratory flow rates $(15,30$ and $60 \mathrm{l} / \mathrm{min}$, particles with diameter $4 \mu \mathrm{m})$ at point $\mathrm{B}$ (placed in trachea $4 \mathrm{~mm}$ off the centreline, $20 \mathrm{~mm}$ above carina, see Fig. 1) are compared in Fig. 6a. All three cases give similar PSD values for low frequencies (up to $30 \mathrm{~Hz}$ ) but higher flow rates produce significantly higher PSD levels in the upper part of the spectra. The PSD is almost constant up to $200 \mathrm{~Hz}$ for $60 \mathrm{l} / \mathrm{min}$ but drops down for $15 \mathrm{l} / \mathrm{min}$ and $30 \mathrm{l} / \mathrm{min}$ reaching only $20-30 \mathrm{~Hz}$. The difference among the different regimes is especially distinct for frequencies higher than $200 \mathrm{~Hz}$. Velocity fluctuations in range $30-300+\mathrm{Hz}$ are therefore responsible for rising TKE with the increase in flow rates. The same behaviour was also found at other measurement positions for both the inspiratory and the expiratory flows. Fig. $6 \mathrm{~b}$ with horizontal axis rescaled using wave number $k=f / \bar{v}$ also shows increased PSD levels at high frequencies for high flow rate which denotes effect of flow rate on energy of small vortices.

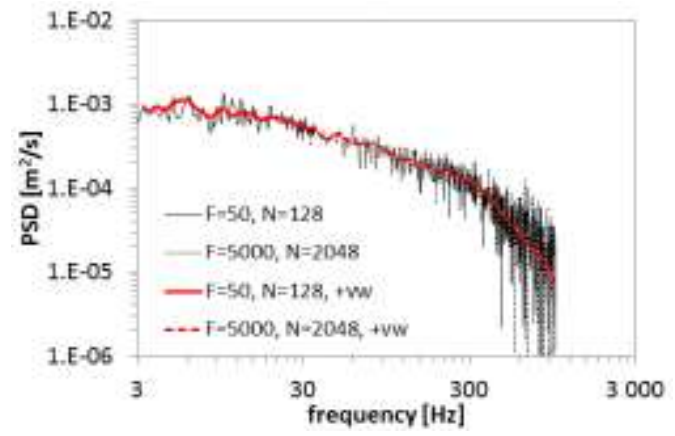

Figure 5. PSD with/without vw, average of three records.
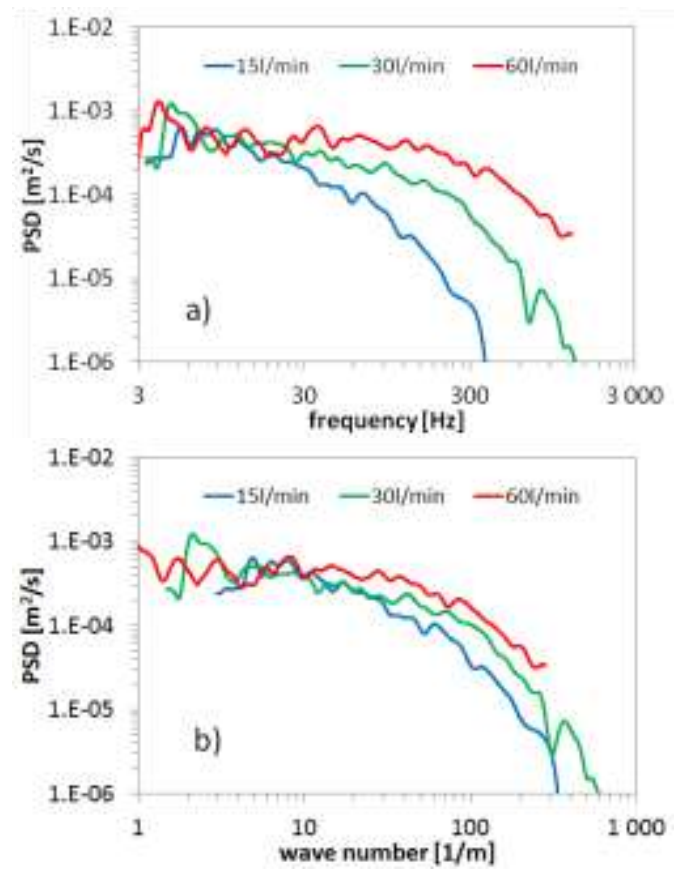

Figure 6. Comparison of different flow regimes.

Particle Size. Steady inspiration flow of particles with 1, 4 and $8 \mu \mathrm{m}$ in diameter was measured at point B at $301 / \mathrm{min}$. No distinct differences in PSDs up to about $500 \mathrm{~Hz}$ (the maximum available correct frequency) are seen in Fig. 7 , except the differences at low frequency part of the spectra due to short record as already mentioned. This result (found also at other measurement positions and for expiration flow) is important and confirms that particles up to $8 \mu \mathrm{m}$ in diameter respond the same way to the vortices at the most energetic frequencies.

Stokes Number. Behaviour of airborne particles of different sizes at various breathing conditions can be explained and discussed by means of Stokes number ( $S t k)$. Stk is a ratio between the particle response time and a characteristic time of the flow field (Crowe et al., 1998). The mean Stk (spatially and temporally averaged) in the case of 


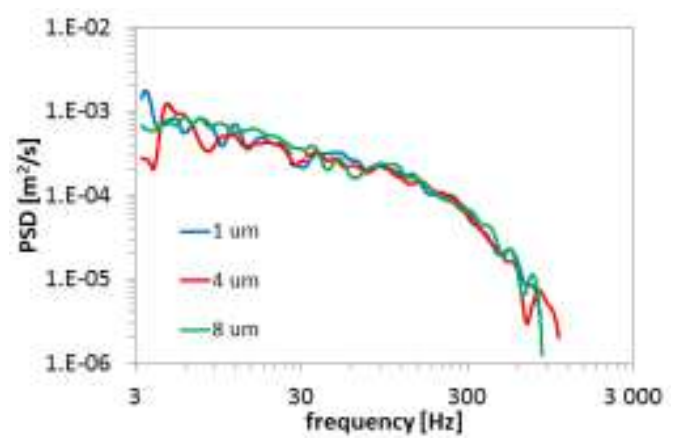

Figure 7. PSD for different particle sizes.

flow in air through a tube of diameter $D$ for a particle having aerodynamic diameter $d_{p}$ and density $\rho_{p}$ is

$$
S t k=\frac{\rho_{p} C_{c} d_{p}^{2} \bar{v}}{18 \mu D}=\frac{4}{18 \pi} \cdot \frac{\rho_{p}}{\mu} \cdot \frac{C_{c} d_{p}^{2} \bar{Q}}{m D^{3}}
$$

where $\mu$ is air dynamic viscosity, $m$ is number of the branches in given branching generation of the model, $\bar{Q}$ is the total mean flow rate, $\bar{v}$ is mean (spatially and temporally) flow velocity and $C_{c}$ is Cunningham correction factor (Theodore, 2005). Mean particle velocity is supposed to be equal to mean flow velocity $\bar{v}$. Stk of the particles in our model falls within the range $0.0002<S t k<0.12$, particular $S t k$ value depends on the branching level, particle size and flow rate (Table 1).

Turbulent flow contains vortices on many different length scales. Let consider a vortical structure with characteristic dimension $D_{v}$. It will produce fluctuations of the particle velocity measured by P/DPA probe at frequency $f=\bar{v} / D_{v}$ with corresponding $S t k_{v}=\rho_{p} C_{c} d_{p}^{2} f / 18 \mu$ (we assume that the air velocity corresponds to the particle velocity if $S t k \ll 1)$. Such defined $S t k_{v}$, contrary to the one given by Eq. (1), does not directly depend on flow regime and position within the lung model. The size of vortices is $2.1,4.2$ and $8.4 \mathrm{~mm}^{7}$ for flow-rates of 15,30 and $60 \mathrm{l} / \mathrm{min}$ respectively at $f=500 \mathrm{~Hz}$ (calculation based on data in Table 1) and corresponding $S t k_{v}$ is 0.002 for $d_{p}=1 \mu \mathrm{m}, 0.028$ for $4 \mu \mathrm{m}$, and 0.1 for $8 \mu \mathrm{m}$ respectively. Such reasonably small $S t k$ suggests that the particles should follow the air flow smoothly at frequencies up to hundreds $\mathrm{Hz}$ as confirmed by our observations (Fig. 7) and other studies (Choi et al., 2010). For such particles, turbulent diffusion could therefore significantly contribute to their deposition.

\footnotetext{
${ }^{7}$ Characteristic size of, for example, Dean vortices in bent tubes (Guan and Martonen, 2000) and vortices produced by mixing of streams from daughter branches (Fresconi and Prasad, 2007) is $\sim 1 / 2$ of the tube diameter. These vortices are in mm scale in trachea and main stem bronchi. Taylor-Görtler-like coherent vortical structures in the supraglottis and the subglottis (Lin et al., 2007) or large horseshoe/hairpin-type vortices (Fresconi et al., 2003) are also mm sized.
}

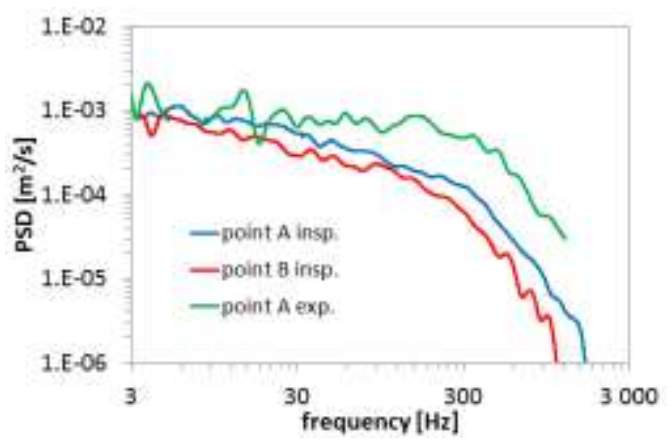

Figure 8. PSD for steady inspiratory and expiratory flows at point $\mathrm{A}$ and for steady inspiratory flow at point B.

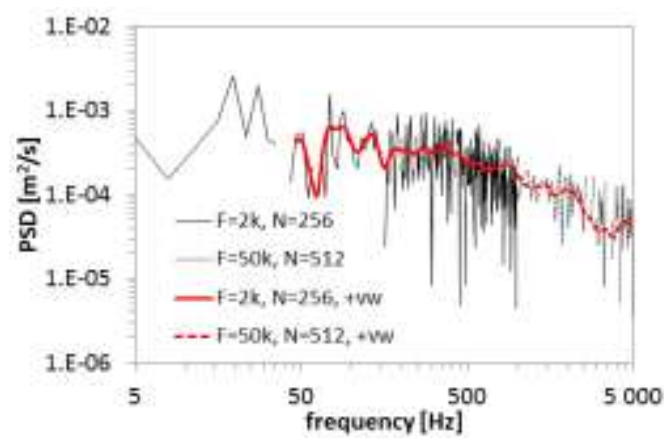

Figure 9. Average PSD for inspiratory cyclic flow at point $\mathrm{C}$.

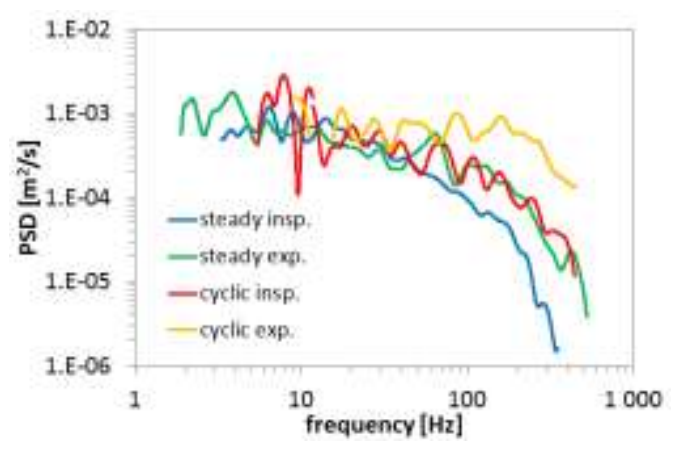

Figure 10. Steady and cyclic insp. and exp. flows.

Other Factors. There is not enough space to describe spatially resolved results in this paper so only an illustrative plot with PSD acquired at two points of the same cross-section (A and B) for inspiration flow at $30 \mathrm{l} / \mathrm{min}$ is shown in Fig. 8. Point $\mathrm{B}$ gives higher intensities of velocity fluctuations in the whole range and mainly at frequencies higher than $200 \mathrm{~Hz}$, but shape of the PSD plot is consistent with the other point. The increased TKE at off-axis position $\mathrm{B}$ could be caused by shear forces (interaction of the fast axial jet with slower near wall air or by asymmetric character of the jet (see Lin et al., 2007). 
Comparison of inspiratory and expiratory flows at point A for $30 \mathrm{l} / \mathrm{min}$ (Fig. 8) shows much higher amount of TKE contained at high frequencies for the expiration flow. This difference can be explained by different way of the turbulence generation which is due to the laryngeal jet flowing into the trachea during inspiration and due to mixing of streams from daughter branches for expiration flow.

\section{Cyclic Flows}

Flow during breathing cycle changes from positive (inspiratory phase) to negative (expiratory phase) and, in the major section of the upper part of the tracheobronchial tree, transition from laminar to turbulent flow occurs with increasing $R e$ (Fig. 2). High-velocity parts of the cycle near inspiration/expiration peaks are the most important for particle deposition. The PSD of cyclic velocity fluctuations was evaluated by applying Kern in a similar way as for the steady flows above on fragments of the breathing cycle considering the flow here as quasi-steady. The PSD estimates acquired such way give only overall information on the average content of TKE in the spectra in the inspected fragment of time. The length of the time window is a compromise to fulfil the quasisteady flow character and to acquire enough data for a reasonable PSD estimate ${ }^{8}$.

Fig. 9 shows such PSD averaged from four records. The data measured at point $\mathrm{C}$ (see Fig. 1) for particle velocity in time window $0.3-0.7 \pi$ (peak inspiration, see Fig. 2) were processed. These data, with mean data rate about $6.7 \mathrm{kHz}$, enabled reliable spectra evaluation up to $1 \mathrm{kHz}$. The short time window $\sim 0.6 \mathrm{sec}$ leads to only 4096 samples in each record and problematic evaluation for frequencies lower than $30 \mathrm{~Hz}$. Large scatter in the PSD spectra and large record-to-record differences were found. The spectrum shows relatively constant values up to $300 \mathrm{~Hz}$. Slow decrease with frequency above $300 \mathrm{~Hz}$ compared to the steady data is seen. Relatively high amplitudes of low frequency fluctuations (below $30 \mathrm{~Hz}$ ) with local minimum at about $40 \mathrm{~Hz}$ are observed here and at other measurement points/regimes. These fluctuations are supposed to be related to large-scale vortices that propagate through the tubes and their influence is recognisable also in time plots of the droplet velocity (Fig. 2), where these vortices lead to deformations of the mean velocity from the original sinusoidal course (more in Jedelsky et al., 2010a). Such character of flow differs from results found for steady breathing - these vortices are typical for cyclic flows.

Exhalation phase of the cyclic flow produces more highfrequency fluctuations than the inspiration flow (Fig. 10) from the same reason as in the steady flow case (Fig. 8).

Corresponding steady and cyclic flows $15 \mathrm{l} / \mathrm{min}$ and 0.51 $\& 4 \mathrm{sec}$ respectively with $4 \mu \mathrm{m}$ particles were compared at point A (Fig. 10). Cyclic flows contain more high-frequency

\footnotetext{
${ }^{8}$ A sort of short-time Fourier transform or Hilbert transform working with non-equidistant sampling would be useful to estimate PSD of velocity fluctuations for individual breathing phases but these are not at disposal yet.
}

velocity fluctuations than the steady flows for both flow directions. PSDs differ from frequencies about $50 \mathrm{~Hz}$ and about half-order difference appears at $100 \mathrm{~Hz}$ already.

\section{Data Rate of LDA Measurements}

The PSD of velocity fluctuations contains interesting information on flow turbulence, which spans over several frequency orders, however quality of the results and upper limit of the frequency range $f_{\max } \cong \dot{n} / 2 \pi$ depends on LDA data rate.

Our CMAG generator produces aerosol with mean concentration $\bar{c}$ in range $10^{4}-10^{6}$ particles $/ \mathrm{cm}^{3}$. After diluting of the aerosol in the mixing system a homogeneous air-particle mixture is delivered into the airway model with $\bar{c} \approx 10^{3}-10^{5}$ particles $/ \mathrm{cm}^{3}$. This dilute aerosol (mean free path in order of hundreds particle diameters) gives a typical data rate of P/DPA measurement in order of $\mathrm{kHz}$. Mean data rate

$$
\dot{n}=\bar{v} \cdot \bar{c} \cdot S \cdot K
$$

where $S$ is area of the measurement volume (mv) projected in the flow direction, $\bar{v}$ is mean particle velocity and yield $K$ depends on visibility of droplets by P/DPA and its validation rate. Laser-Doppler based techniques are capable to measure only one particle at given time; if the system detects a particle, then other particles appearing in the $\mathrm{mv}$ at the same time or flowing in before the first one exits the mv will be rejected from evaluation. The theoretical maximum measurement frequency would be (assuming equidistant sampling for simplification):

$$
\dot{\bar{n}}_{\max } \cong 1 / \Delta T=v / a=v \cdot \cos \theta / D_{1}
$$

where size $a$ of the mv along the flow direction depends on the angle between incident laser beams $\theta$ and on their diameter, $D_{l} . \Delta T$ is particle residence time in the mv. Eq. (3) is valid for particle diameter $d_{p} \ll D_{l}$. The $\mathrm{mv}$ is defined by the intersection of two Gaussian laser beams. This volume in the shape of an ellipsoid is seen by receiving optics through a slit of width $s$ which reduces its length. Resulting mv has roughly the shape of an oblique cylinder with lengths of main halfaxes $a / 2$ and $D_{l} /(2 \cos \theta)$ and height $s$. The increase in slit size enlarges the projection area of mv into the flow direction, which is approximately $S=s \cdot d / \cos \varphi$ where $\varphi$ is scattering angle and thereby increases data rate according to Eq. (2). It however also reduces spatial resolution of the measurement. Variation in the laser beam diameter $D_{l}$ influences $S$ and therefore changes number of detected particles with interparticle arrival time gaps that are larger than $\Delta T$. Change in size $a$ would reduce $\dot{\bar{n}}_{\text {max }}$ (Eq. (3)). For instance doubling $D_{l}$ will also double up detected particle number $\mathrm{p}(\Delta t)$ and simultaneously will twice reduce corresponding interparticle arrival time $\Delta t$ as seen at Fig. 11. A simulation made for our 


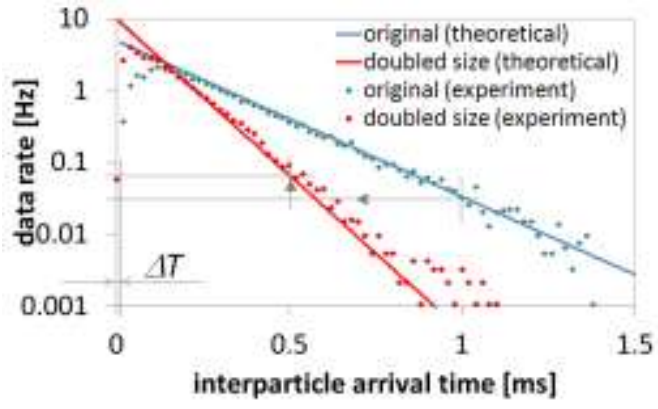

Figure 11. Frequency plot of arrival time between two particles for $1 \mu \mathrm{m}$ particles and flow rate $30 \mathrm{l} / \mathrm{min}$ at point A.

setup $\left(D_{l 0}=0.27 \mathrm{~mm}, s=0.3 \mathrm{~mm}, k=0.9, \theta=11.4^{\circ}, \varphi=\right.$ $50^{\circ}, c=10^{4} \mathrm{~cm}^{-3}, \bar{v}=3 \mathrm{~m} / \mathrm{s}$, which leads to $\dot{\bar{n}}=3.4 \mathrm{kHz}$ according Eq. (2)) has shown optimum $D_{l}=1.3 D_{l 0}$ with increase in data rate by about $10 \%$.

Particle concentration is limited by the particle source used and by desired character of the flow. Higher concentration is generally better, but the two-phase mixture would change from dilute to dense with increase in the concentration which could affect the particle flow. The yield $K$ is given by the measurement system setup and its technical condition. Also monodispersity of the seeding particles could have an effect. The detector sensitivity of a system measuring polydisperse aerosol must be set to a value at which large particles will not saturate the detector yet and, in such case, small particles might not meet detection limit.

\section{CONCLUSIONS}

Extensive data on particle flow in time domain are contained in P/DPA results acquired in steady and cyclic flows in realistic airway model. The possibility of estimation of the velocity fluctuation PSD from these data was presented. Kern software was used to calculate the PSDs and optimised for our data. Similar fundamental character of TKE spectra was found for all processed data with systematic quantitative differences for (1) inspiration and expiration flows, (2) different positions in the airway tubes and (3) different flow regimes.

The amplitude of velocity fluctuations in PSD representation is relatively constant up to certain frequency ( $30-50 \mathrm{~Hz}$ depending on the flow regime), followed by moderately decreasing trend (up to $\sim 300 \mathrm{~Hz}$ for steady and $~$ $500 \mathrm{~Hz}$ for cyclic flows) and more rapid drop afterwards. The PSD curves are generally smooth with no distinct peaks identified in any analysed main-stream data file.

The PSD in the inspected frequency range $(\sim 2-700 \mathrm{~Hz})$ is practically independent of the particle size within $1-8 \mu \mathrm{m}$ in whole range of flow regimes under consideration. This important finding, explained by low $S t k$ values for observed cases, implies that micron sized aerosol particles closely follow the air flow. Particle P/DPA data can therefore be used for estimation of time-resolved local air velocity and for description of air flow structures in the airways. Further conclusion is that for these low Stk particles turbulent diffusion could significantly contribute to their deposition on the airway walls.

Successfulness of PSD estimation depends on available data. Present P/DPA results with mean data rate $0.5-10 \mathrm{kHz}$ (depending on position, particle size and flow conditions) allow for maximum usable frequency $0.5-1.6 \mathrm{kHz}$. The most energetic fluctuations (energy-containing range + inertial subrange of the spectra) are included within such spectra but information on high-frequency fluctuations (dissipation range) can be hardly resolved. High concentration aerosol, larger measurement volume and optimum and precise LDA setup are crucial for an increase of the frequency range of PSD estimations. Improved results for velocity fluctuations could be used for validation of LES and DNS predictions where more effective turbulence models are to be developed (Longest and Holbrook, 2011).

\section{ACKNOWLEDGEMENTS}

This work was supported by projects ME 09030 of the program KONTAKT and OC10052, COST Action Particles No. MP0806 funded by the Ministry of Education, Youth and Sports of the Czech Republic, by project GA 105/11/1339 funded by the Czech Science Foundation and by the project FSI-S-11-6 of the Brno University of Technology.

\section{REFERENCES}

Adler, K., and Brücker, C., 2007, "Dynamic flow in a realistic model of the upper human lung airways", Experiments in Fluids, Vol. 43, no. 2-3, pp. 411-423.

Adrian, R. J. and Yao, C. S., 1987, "Power spectra of fluid velocities measured by laser Doppler velocimetry", Exp. Fluids 5, 17-28.

Azarmi, S., Roa, W. H., and Löbenberg, R., 2008, "Targeted delivery of nanoparticles for the treatment of lung diseases", Advanced Drug Delivery Reviews, Vol. 60, pp. 863875 .

Benedict, L. H., Nobach, H., and Tropea, C., 2000, "Estimation of turbulent velocity spectra from laser Doppler data", Meas. Sci. Technol. 11, 1089.

Chan, T. L., Schreck, R. M., and Lippmann, M., 1980, "Effect of the laryngeal jet on particle deposition in the human trachea and upper bronchial airways", Journal of Aerosol Science, Vol. 11, No. 5-6, pp. 447-459.

Cheng, K. H., Cheng, Y. S., Yeh, H. C., and Swift, D. L., 1997, "An Experimental Method for Measuring Aerosol Deposition Efficiency in the Human Oral Airway", Am. Ind. Hyg. Assoc. J. 58, pp. 207 - 213.

Choi, Y., Park, Y., and Lee, Ch., 2010, "Helicity and geometric nature of particle trajectories in homogeneous isotropic turbulence", International Journal of Heat and Fluid Flow, Vol. 31, No. 3, pp. 482-487. 
Cohen, B. S., Sussman, R. G., and Lippmann, M., 1993, "Factors affecting distribution of airflow in a human tracheobronchial cast", Respiration physiology, Vol. 93, no. 3, pp. 261-278.

Corcoran, T. E., and Chigier, N., 2000, "Characterization of the laryngeal jet using Phase Doppler Interferometry", J. of Aerosol Medicine, Vol. 13, No. 2, pp. 125-137.

Corieri, P., and Riethmuller, M. L., 1989, "Laser Doppler velocimetry and computer automation to measure low velocities in a pulmonary model", ICIASF Record, Int. Congress on Instrumentation in Aerospace Simulation Facilities, pp. 226-236.

Crowe, C. T., Sommerfeld, M., Tsuji, Y., 1998, "Multiphase flows with droplets and particles", CRC Press, New York.

Fresconi, F. E., and Prasad, A. K., 2007, "Secondary velocity fields in the conducting airways of the human lung", Journal of Biomechanical Engineering, Vol. 129, no. 5, pp. 722-732.

Fresconi, F. E., Wexler, A. S., and Prasad, A. K., 2003, "Expiration flow in a symmetric bifurcation", Experiments in Fluids, Vol. 35, no. 5, pp. 493-501.

Gemci, T., Corcoran, T. E., and Chigier, N., 2002, "A numerical and experimental study of spray dynamics in a simple throat model", Aerosol Science and Technology, Vol. 36, No. 1, pp. 18-38.

Große, S., Schröder, W., Klaas, M., Klöckner, A., and Roggenkamp, J., 2007, "Time resolved analysis of steady and oscillating flow in the upper human airways", Experiments in Fluids, Vol. 42, No. 6, pp. 955-970.

Guan, X., and Martonen, T. B., 2000, "Flow transition in bends and applications to airways", Journal of Aerosol Science, Vol. 31, No. 7, pp. 833-847.

Jedelsky, J., Lizal, F. and Jicha, M., 2009, "Realistic transparent human airway model: flow distribution and aerosol transport under steady and unsteady flows". In Proceedings of the $20^{\text {th }}$ Int. Symp. on Transport Phenomena, Victoria, Canada, $7 \mathrm{p}$.

Jedelsky, J., Lizal, F., and Jicha, M., 2010a, "Some Aspects of Particle Motion under Cyclic Flow in Realistic Human Airway Model", In Proceedings of the $21^{\text {st }}$ ISTP, Kaohsiung, Taiwan, pp. 1-8, ISBN 978-986-6184-25-3.

Jedelsky, J., Lizal, F., and Jicha, M., 2010b, "Particle Motion in a Realistic Human Airway Model under Steady and Cyclic Flows", in proceedings XXIV. Symposium on Anemometry, Prague, ASCR, pp. 1-9.

Kleinstreuer, C., and Zhang, Z., 2003, "Laminar-toturbulent fluid-particle flows in a human airway model", International Journal of Multiphase Flow, Vol. 29, 271-289.

Kleinstreuer, C., Zhang, Z., and Li, Z., 2008, "Modeling airflow and particle transport/deposition in pulmonary airways", Respiratory Physiology \& Neurobiology, Vol. 163, pp. 128-138.

Li, Z., Kleinstreuer, C., and Zhang, Z., 2007, "Simulation of airflow fields and microparticle deposition in realistic human lung airway models. Part I: Airflow patterns",
European Journal of Mechanics B/Fluids, Vol. 26, pp. 632649.

Lieber, B. B., and Zhao, Y., 1998, "Oscillatory flow in a symmetric bifurcation airway model", Annals of Biomedical Engineering, Vol. 26, No. 5, pp. 821-830.

Lin, C., Tawhai, M. H., McLennan, G., and Hoffman, E. A., 2007, "Characteristics of the turbulent laryngeal jet and its effect on airflow in the human intra-thoracic airways", Respiratory Physiology and Neurobiology, Vol. 157, No. 2-3, pp. 295-309.

Lizal, F., Elcner, J., Hopke, P. K., Jedelsky, J., Jicha, M., 2011, "Development of a realistic human airway model". Proceedings of the Institution of Mechanical Engineers, Part H: Journal of Engineering in Medicine 0954411911430188, first published on December 16, 2011 doi: 10.1177/0954411911430188.

Longest, P. W., and Holbrook, L. T., 2011, "In silico models of aerosol delivery to the respiratory tract Development and applications", Advanced Drug Delivery Reviews, in print.

Martonen, T., Quan, L., Zhang, Z., and Musante, C., 2002, "Flow simulation in the human upper respiratory tract", Cell Biochemistry and Biophysics, Vol. 37, pp. 27-36

Nobach H., Müller E., and Tropea C., 1996, "Refined Reconstruction Techniques for LDA Data Analysis", Proc. of the 8th International Symposium on Applications of Laser Techniques to Fluid Mechanics, Lisbon, Portugal.

Nobach, H., 2002, "Local time estimation for the slotted correlation function of randomly sampled LDA data", Experiments in Fluids, Vol. 32, pp. 337-345.

Nobach, H., 2006, "Data for comparison of spectra measured by CTA and LDA techniques", http://ldvproc.nambis.de/data/dtudata.html, (accessed 2011).

Obrist, D., Henniger, R., and Kleiser, L., 2011, "Subcritical spatial transition of swept Hiemenz flow", in TSFP7 - 7th Int. Symp. on Turbulence and Shear Flow Phenomena, Ottawa, Canada, 2011.

Ramuzat, A., and Riethmuller, M. L., 2002, "PIV investigations of oscillating flows within a 3D lung Multiple Bifurcation model". 11th Int. Symp. On Appl. of Laser Techniques to Fluid Flows, Lisbon, Portugal, paper 19-1.

Schmidt, A., Zidowitz, S., Kriete, A., Denhard, T., Krass, S. and Peitgen, H. O., 2004, "A digital reference model of the human bronchial tree", J. Computerized Medical Imaging and Graphics, Vol. 28, pp. 203-211.

Scott, P. F., 1974, "Random sampling theory and its application to laser velocimeter turbulent spectral measurements", Report 74CRD216, General Electric Co, Corporate Research and Development.

Sosnowski, T. R., Moskal, A., and Gradon, L., 2007, "Mechanims of Aerosol Particle Deposition in the OroPharynx Under Non-Steady Airflow, " Ann. Occup. Hyg., Vol. 51, No. 1, pp. 19-25.

Su, W. C. and Cheng, Y. S., 2009, "Deposition of Manmade Fibers in Human Respiratory Airway Casts", J. Aerosol Sci., Vol. 40(3), pp. 270-284. 
Tanaka, G., Ogata, T., Oka, K., and Tanishita, K., 1999, "Spatial and temporal variation of secondary flow during oscillatory flow in model human central airways", Journal of Biomechanical Engineering, Vol. 121, No. 6, pp. 565-573.

Theodore, L., 2005, Nanotechnology: Basic Calculations for Engineers and Scientists, Wiley-Interscience, ISBN: 0471739510.

Theunissen, R., and Riethmuller, M. L., 2008, "Particle image velocimetry in lung bifurcation model", Topics in Applied Physics, Vol. 112, pp. 73-101.

Tropea, C., Nobach, H., Ramond, A., and Reulet, P., 2001, "Turbulent Velocity Spectra from Laser Doppler Data", Proc. 3rd ONERA - DLR Aerospace Symposium, Paris, France.

Tummers, M. J., and Passchier, D. M., 1996, "Spectral estimation using a variable window and the slotting technique with local normalization", Meas. Sci. Technol. 71541.

Zhang, Z., Kleinstreuer, C., and Kim, C. S., 2001, "Flow structure and particle transport in a triple bifurcation airway model". Journal of Fluids Engineering, Trans ASME, Vol. 123, pp. 320-330.

Zhang, Z., and Kleinstreuer, C., 2002, "Transient airflow structures and particle transport in a sequentially branching lung airway model", Phys. Fluids, Vol. 14, No. 2.

Zhang, Z., and Kleinstreuer, C., 2004, "Airflow structures and nano-particle deposition in a human upper airway model", Journal of computational physics, Elsevier, Amsterdam, Vol. 198, No. 1, pp. 178-210. 